\title{
Expanded SEIRCQ Model Applied to COVID-19 Epidemic Control Strategy Design and Medical Infrastructure Planning
}

\author{
J. M. V. Grzybowski $\mathbb{D}^{1},{ }^{1}$ R. V. da Silva $\mathbb{D}^{1},{ }^{1}$ and M. Rafikov ${ }^{2}$ \\ ${ }^{1}$ Environmental Science and Technology Postgraduate Program (PPGCTA), Federal University of Fronteira Sul (UFFS), Erechim, \\ Rio Grande do Sul, Brazil \\ ${ }^{2}$ Center for Engineering, Modeling and Applied Social Sciences (CECS), Federal University of ABC (UFABC), Santo André, \\ São Paulo, Brazil \\ Correspondence should be addressed to J. M. V. Grzybowski; jose.grzybowski@uffs.edu.br
}

Received 21 April 2020; Revised 22 June 2020; Accepted 27 June 2020; Published 8 August 2020

Academic Editor: Ivan Giorgio

Copyright $\odot 2020$ J. M. V. Grzybowski et al. This is an open access article distributed under the Creative Commons Attribution License, which permits unrestricted use, distribution, and reproduction in any medium, provided the original work is properly cited.

\begin{abstract}
The rapid spread of COVID-19 has demanded a quick response from governments in terms of planning contingency efforts that include the imposition of social isolation measures and an unprecedented increase in the availability of medical services. Both courses of action have been shown to be critical to the success of epidemic control. Under this scenario, the timely adoption of effective strategies allows the outbreak to be decelerated at early stages. The objective of this study is to present an epidemic model specially tailored for the study of the COVID-19 epidemics, and the model is aimed at allowing the integrated study of epidemic control strategies and dimensioning of the required medical infrastructure. Along with the theoretical model, a case study with three prognostic scenarios is presented for the first wave of the epidemic in the city of Manaus, the capital city of Amazonas state, Brazil. Although the temporary collapse of the medical infrastructure is hardly avoidable in the state-of-affairs at this time (April 2020), the results show that there are feasible control strategies that could substantially reduce the overload within reasonable time. Furthermore, this study delivers and presents an intuitive, straightforward, free, and open-source online platform that allows the direct application of the model. The platform can hopefully provide better response time and clarity to the planning of contingency measures.
\end{abstract}

\section{Introduction}

The rapid spread of COVID-19 outbreak has become a very challenging situation due to its substantial demand for medical infrastructure. The lack of knowledge about the virus at early stages severely limits the effectiveness of available clinical treatments and thus contributes to longer hospitalization periods that might nevertheless result in the loss of a considerable share of patients, as shown in recent clinical publications [1-4]. As largely explained by political and medical authorities from several countries and the WHO (World Health Organization), the main problem of having a steep epidemic curve is the overload it might cause on health care infrastructure [5]. In this sense, not only medical materials and equipment but also medical personnel suddenly become insufficient, who become especially prone to infection when subjected to an overload of working hours and lack of adequate personal protection equipment [5].

As the world faces the consequences of a largely unknown infectious agent that spreads exponentially, the timely adoption of adequate control and mitigation strategies is of utmost importance. In this context, the mathematical modelling of epidemic dynamics can provide a valuable contribution. Beyond helping to unveil prognostics of future behavior under different scenarios that are unaccessible otherwise, it allows the application of numerical parameters from previous studies and clinical expertise (see, for instance, [1-4]). Furthermore, while the focus of epidemic modelling is control strategies, the estimated epidemic timeline can help forge ways to optimize the 
maintenance of economic activities to their fullest yet below levels that are detrimental to epidemic control. Finding this tenuous and fragile equilibrium between epidemic control and economic activity is at the core of several debates [6]. In this sense, mathematical models can provide a rather solid basis for discussion, while their underlying assumptions can be openly scrutinized and updated to evaluate scope and accuracy of the resulting conclusions. Another factor that has gained importance as authorities acknowledged the lack of infrastructure to fight the epidemic is time. If countries can gain time by adopting adequate strategies, they can prepare better to properly handle the epidemic. As such, the study and evaluation of epidemic models can not only provide future scenarios that allow the dimensioning of the infrastructure but also test strategies to flatten the infection curve as much as needed to fit the achievable capacity of the health care system [7-9].

Following the seminal works on epidemic dynamics by Kermack and McKendrick [10-12], many epidemic models were proposed and studied in the literature, including but not limited to SIR [13, 14], SIS [14], SIRS [14, 15], SEIR $[16,17]$, SEIS $[18,19]$, and MSEIR [13]. Among those, the SEIR model following classical assumptions is a rather interesting starting point to the study of the COVID-19 since it is a rather simple model for which a substantial body of literature is available. The SEIR model consists of four population subclasses: S (susceptible), E (exposed, which are infected but not infective), I (infected and infective), and $\mathrm{R}$ (removed). The compartment $\mathrm{R}$ encompasses both recovered and deceased. The recovered individuals in this compartment represent the fact that once COVID-19 patients recover, they are considered to have built lasting resistance to the infection, at least to the timeframe of the first wave of the epidemic. The SEIR model was used as basis in recent studies about COVID-19 [7,9]. To allow the implementation of epidemic control strategies and dimensioning of medical infrastructure under different scenarios, the model presented in this study was expanded with new compartments.

The objective of this study is to present an epidemic model tailored for COVID-19, and the model is aimed at allowing the integrated study of epidemic control strategies and dimensioning of the medical infrastructure required in different scenarios. The model is an expanded version of the well-known SEIR model, in which two new compartments are added: confinement of susceptible (C) and quarantine of infected (Q). These compartments are aimed at allowing the implementation of two of the epidemic control measures that also include social distancing and vaccination, the latter as a future prospect in the case this scenario extends for a long period. Furthermore, three equations that account severe and critical hospitalization cases and the number of deaths are included to give an account of the burden on health care infrastructure. To ease the application of the model by a general audience, we provide an implementation of the expanded SEIRCQ model as an intuitive, straightforward, free, and open-source online platform (https:// dashseir.herokuapp.com/) that allows the direct application of the model with clinical and physical parameters that are accessible in the literature. To illustrate the application of the model, we also provide a complete case study for the city of Manaus, the capital city of Amazonas State, Brazil. Manaus shall face the collapse of the medical care capacity due the first wave of the coronavirus outbreak [20]. We use the model to evaluate the current state-of-affairs and show that they shall lead the infection curve to overwhelm the current medical care capacity for several months in case they remain unchanged. Then, we test possible measures and strategies to mitigate the hazardous effects of the epidemic. As such, the justification for this manuscript is twofold: to introduce the rationale behind the working of the platform and to serve as a reference for its application.

\section{Literature Review}

2.1. Epidemic Models. The study of epidemic models dates to 1927 with a set of three publications by Kermack and McKendrick, which were more recently republished in digital versions [10-12]. Since then, a number of models were proposed and studied, such as SIR [13, 14], SIS [14], SIRS [14, 15], SEIR [16, 17], SEIS [18, 19], and MSEIR [13], among others, which are known as compartmental models. The general idea is that the population is subdivided into compartments, each governed by a dynamical equation. The equations can be either deterministic or stochastic, and each compartment can be further stratified by gender, age, or any other feature that is regarded as relevant for a given study. Furthermore, the model can be either spatially homogeneous or heterogeneous, depending on the relevance of location to the dynamics of the epidemic.

Several models have been proposed to describe the dynamics of the COVID-19 epidemic. Naturally, each modelling approach seeks to describe a set of variables of interest according to specific objectives. A fair share of the recent models appearing in the literature for the study of COVID-19 are extensions of the well-known SEIR (susceptible-exposedinfected-removed) model by Li and Muldowney [17], Roda et al. [9], López and Rodó [21], and Kuniya [7].

A study by Lin et al. [22] took as basis the SEIR model and expanded it by including extracompartments that were aimed at modelling the public perception of risk and the cumulative number of cases (both reported and not reported). In fact, the results presented in $\mathrm{Wu}$ and McGoogan [23] suggest that a considerable share of nonreported cases exist. Thus, modelling the cumulative number of cases, as done by Lin et al. [8], is likely to produce more realistic scenarios and prognostics. Furthermore, a step-like function is applied to model the zoonotic transmission at early stages, from which it was possible to estimate the approximate number of zoonotic infections that presumably occurred in Wuhan and trace the epidemic back to its origin. To deal with the lack of a long enough time series for COVID-19, Lin et al. [8] applied parameters from the influenza epidemic from 1918 in London, UK [24], to fit the model to the official data from Wuhan, China. The main difficulty associated with the application of this model is the definition of control strategy parameters (such as governmental action strength) since they cannot be straightforwardly mapped into collectible real-world parameters. 
Roda et al. [9] showed that the confirmed-case data are the main reason to the wide range of variations in model predictions for the COVID-19 epidemic using mathematical models, which pose a difficulty when estimating real transmission rates, since transmission rate coefficients in model calibration might have a very wide range. Roda et al. [9] also point that the confirmed-case data represent only a fraction of the total infected population. Thus, fitting the model to confirmed-case data is likely to produce misleading prognostics of future scenarios in terms of the peak values.

2.2. COVID-19Epidemic Parameters. The knowledge gained during the crisis in China provides several useful parameters for modelling. For instance, the work by $\mathrm{Wu}$ and McGoogan [23] considered over 72, 000 cases from the first wave of the epidemic in China and established that $81 \%$ of the cases were mild (all the way from asymptomatic to mild symptoms), 14\% were severe (high respiratory frequency, low blood oxygen saturation, and lung infiltrates), and 5\% were critical (respiratory failure, septic shock, and/or multiple organ dysfunction or failure). Furthermore, the authors also found the overall case-fatality rate of $2.3 \%$, while the death rate was $49 \%$ among critical patients, and no deaths were reported in mild and severe cases. Wu and McGoogan [23] also presented numbers that hint that insufficient testing may have caused case-fatality rates to be overestimated approximately by 7-fold factor. Interestingly, a 7-fold factor was also found to be the factor of subnotification of infected individuals in the State of Rio Grande do Sul, Brazil, as concluded the first large field study based on massive testing of individuals [25]. The basic reproduction number in Japan considering the SEIR model was estimated by Kuniya [7] as $R_{0}=2.6$ (2.4 to 2.8 in a $95 \%$ confidence interval), which means that an infected individual will infect, on average, 2.6 new individuals. Meanwhile, considering the case of Wuhan, Tang et al. [26] found that the basic reproduction number can be as large as 6.47 (5.71 to $7.23,95 \% \mathrm{CI})$ and $\mathrm{Li}$ et al. [22] found 2.2 (1.4 to 3.9, 95\% CI) on the basis of analysis of 425 laboratory-confirmed cases. Regarding the basic reproduction number after social distancing measures, Wang et al. [3] considered a scenario with $R_{m}=0.9$, which later was found to provide an accurate description of the epidemic dynamics after the social distancing measures were implemented in China.

As other important parameters to estimate the load upon medical care services, Chen et al. [27] calculated the average duration of hospitalization of 31 discharged patients and found 12.39 days ( $\pm 4.77,95 \% \mathrm{CI}$ ). Meanwhile, Li et al. [22] calculated the average incubation period to be 5.2 days (4.1 to $7.0,95 \% \mathrm{CI}$ ), while $\mathrm{Bi}$ et al. [1] found 4.8 days ( 4.2 to 5.4 , $95 \% \mathrm{CI}$ ) on the basis of 391 cases and 1,286 of their close contacts. To take advantage of the results from clinical studies, the next section presents the expanded SEIRCQ model in a formulation that allows explicit application of clinical and physical parameters. This is aimed at allowing straightforward application of medical literature about COVID-19.

\section{Materials and Methods}

This section gives an account of the proposed model and the epidemic control strategies to be applied. It also presents the region considered in the case study and the methods applied to acquire the model parameters.

3.1. The Expanded SEIRCQ Model. Consider a population with $N$ individuals and the following stratification compartments: susceptible $(S)$, exposed $(E)$, infected $(I)$, removed $(R)$, quarantined infected $(Q)$, and confined susceptible $(C)$, which are represented in Figure 1 and related according to the equations:

$$
\begin{aligned}
& \frac{\mathrm{d} S}{\mathrm{~d} t}=-\frac{R_{t}}{T_{\mathrm{INF}}} \frac{S I}{N}-R_{\mathrm{CNF}} S+\frac{1}{T_{\mathrm{CNF}}} C-\frac{R_{\mathrm{VCN}}}{T_{\mathrm{IMN}}} S, \\
& \frac{\mathrm{d} E}{\mathrm{~d} t}=\frac{R_{t}}{T_{\mathrm{INF}}} \frac{S I}{N}-\frac{1}{T_{\mathrm{INC}}} E, \\
& \frac{\mathrm{d} I}{\mathrm{~d} t}=\frac{1}{T_{\mathrm{INC}}} E-\frac{R_{\mathrm{QRT}}}{T_{\mathrm{LIC}}} I-\frac{1}{T_{\mathrm{INF}}} I, \\
& \frac{\mathrm{d} R}{\mathrm{~d} t}=\frac{1}{T_{\mathrm{INF}}} I+\frac{R_{\mathrm{VCN}}}{T_{\mathrm{IMN}}} S+\frac{1}{T_{\mathrm{QRT}}} \mathrm{Q}, \\
& \frac{\mathrm{d} C}{\mathrm{~d} t}=R_{\mathrm{CNF}} S-\frac{1}{T_{\mathrm{CNF}}} C, \\
& \frac{\mathrm{d} Q}{\mathrm{~d} t}=\frac{R_{\mathrm{QRT}}}{T_{\mathrm{LIC}}} I-\frac{1}{T_{\mathrm{QRT}}} \mathrm{Q},
\end{aligned}
$$

where $R_{t}$ is the average number of new infections caused by an infected individual, $T_{\mathrm{INF}}$ is the average duration of the infection in an individual, $R_{\mathrm{CFN}}$ is the rate of confinement of susceptible, $T_{\mathrm{CNF}}$ is the average time of confinement of a susceptible individual, $R_{\mathrm{VCN}}$ is the rate of vaccination of susceptible individuals, $T_{\text {IMN }}$ is the average time for immunization of an individual after vaccination, $T_{\text {INC }}$ is the average incubation time of an individual exposed to the infection, $T_{\mathrm{INC}} R_{\mathrm{QRT}}$ is the rate of infected individuals entering quarantine, $T_{\text {LIC }}$ is the average time lag until an infected individual is put in quarantine, and $T_{\mathrm{QRT}}$ is the average time of quarantine. As a result, the total population is given by

$$
N=S+E+I+R+C+Q \text {. }
$$

The parameter $R_{t}$ can be a function of time to describe the strategy of lockdown, during which the infection rate tends to be lower. For instance, to describe the transition from free circulation to isolation at time $T_{\text {ISO }}$, one can define $R_{t}$ as a function defined by parts as

$$
R_{t}= \begin{cases}R_{0}, & t \leq T_{\mathrm{ISO}} \\ R_{m}, & t>T_{\mathrm{ISO}}\end{cases}
$$

where $R_{t}=R_{0}$ during $\left(-\infty, T_{\text {ISO }}\right]$ and $R_{t}=R_{m}$ from $T_{\text {ISO }}$ on. In this case, it is expected that $R_{0}>R_{m}$. 


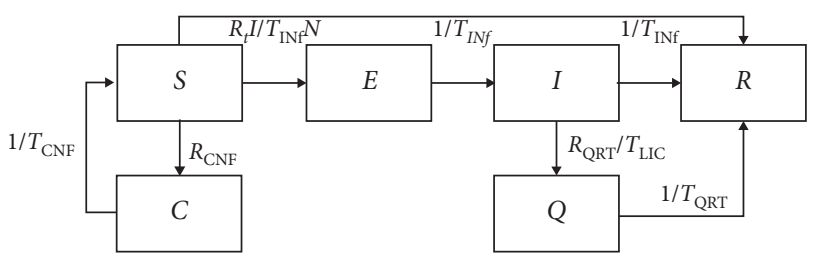

FIGURE 1: Flowchart of the SEIRCQ model.

It is supposed that a portion of the infected population will need hospitalization and a smaller portion will require hospitalization in an Intensive Care Unit (ICU). This portion of the infected population causes greater concern due to the prospect of overload in the health care infrastructure. To model the dynamics of individuals requiring hospitalization and individuals requiring intensive care, the model is expanded and two new state variables are defined, $H$ and $U$, respectively. The rates of change in the number of individuals requiring hospitalization and those requiring intensive care are modeled as

$$
\begin{gathered}
\frac{\mathrm{d} H}{\mathrm{~d} t}=\frac{R_{\mathrm{HSP}}}{T_{\mathrm{INC}}} E-\frac{1}{T_{\mathrm{HSP}}} H, \\
\frac{\mathrm{d} U}{\mathrm{~d} t}=\frac{R_{\mathrm{ICU}}}{T_{\mathrm{INC}}} E-\frac{1}{T_{\mathrm{ICU}}} U,
\end{gathered}
$$

where $R_{\mathrm{HSP}}$ is the rate of hospitalization in simple hospital beds, $T_{\text {HSP }}$ is the average duration of hospitalization in simple hospital beds, $R_{\mathrm{ICU}}$ is the rate of critical cases requiring hospitalization in ICUs, and $T_{\text {ICU }}$ is the average duration of hospitalization in ICUs. In any case, we consider valid the inequality $1 \ll R_{\mathrm{HSP}} \ll R_{\mathrm{ICU}}$, which represents the fact that the number of hospitalizations in common beds is a fraction of the current number of infected individuals, and that the number of hospitalizations in ICU beds is a fraction of the hospitalizations. Besides, the number of mild cases (that do not require hospitalization) can be represented by $M$ and defined as

$$
M=I-H-U
$$

To assess the possible number of deaths in different scenarios, the number of deceased individuals is defined by $P$. The number of fatal cases is modeled as a fraction of those who require intensive treatment. Note that, in practice, not all patients who require intensive treatment can access it since the number of ICU beds is limited. In the model, we will express this limitation by $N_{\text {ICU }}$, which represents the number of ICU beds available to COVID-19 patients in this system. Thus, the rate of change in the number of deaths considers two strata of patients who need an ICU: those who have access and those who cannot, each with a different mortality rate. It is expected that the patient who needs intensive care and does not obtain access will have lesser chances of survival as compared to the patient who needs intensive care and obtains access. Thus, two mortality rates are defined, $R_{\mathrm{DHT} 1}$ and $R_{\mathrm{DHT} 2}$, for patients with access and without access to ICU, respectively. The rate of change in the number of deaths is modeled as

$$
\frac{\mathrm{d} P}{\mathrm{~d} t}=\frac{R_{\mathrm{DHT} 1}}{T_{\mathrm{ICU}}} \bar{U}+\sigma \frac{R_{\mathrm{DHT} 2}}{T_{\mathrm{FOR}}}\left(U-N_{\mathrm{ICU}}\right),
$$

where $\bar{U}$ represents the number of patients in need of intensive care and obtains access to an ICU bed. The variable $\bar{U}$ receives the value of the state variable $U$ up to the limit of ICU vacancies, $N_{\text {ICU }}$, according to function

$$
\bar{U}= \begin{cases}U, & U \leq N_{\mathrm{ICU}}, \\ N_{\mathrm{ICU}}, & U>N_{\mathrm{ICU}} .\end{cases}
$$

In addition, $T_{\text {FOR }}$ defines the average survival time for a patient who needs intensive treatment but cannot access an ICU bed. In this way, a fraction $R_{\mathrm{DHT} 2}$ of critical patients not able to find an ICU are removed from $U$ and transferred to compartment $P$ after a period of $T_{\text {FOR }}$. Meanwhile, $\sigma$ is a function that takes on binary values and controls the second term of equation (6). When $U$ is greater than the number of ICU beds available, the function returns a unit value and the second term of the equation comes into play with its respective mortality rate $R_{\mathrm{DHT} 2}$ and mean survival time $T_{\mathrm{FOR}}$. The mortality according to the increased rate $R_{\mathrm{DHT} 2}$ is modeled as proportional to the number of people in need of intensive treatment but does not get access to a bed due to overcrowding. Thus, the $\sigma$ function is modeled as

$$
\sigma=\left\{\begin{array}{l}
0, U \leq N_{\mathrm{ICU}} \\
1, U>N_{\mathrm{ICU}}
\end{array}\right.
$$

\subsection{Epidemic Control Strategies Using the SEIRCQ Model.} The SEIRCQ model allows the application of four epidemic control strategies. The aim of this section is to present the rationale under each of them and define measures of effectiveness for each one separately and then to all of them combined.

3.2.1. Quarantine of Infected Individuals. The strategy of quarantine of infected individuals seeks to remove from circulation those who are infected to neutralize their contribution to the spread of the infection. By decreasing the number of circulating infected, $I$, one has the multiplication $R_{t} S I$ assume lower values, such that the rate of change in the number of individuals exposed to the infection, given as

$$
\frac{\mathrm{d} E}{\mathrm{~d} t}=\frac{R_{t}}{T_{\mathrm{INF}}} \frac{S I}{N}-\frac{1}{T_{\mathrm{INC}}} E,
$$

decreases accordingly. To have the epidemic under control, it is necessary that the number of circulating infected individuals decreases, i.e.,

$$
\frac{\mathrm{d} I}{\mathrm{~d} t}=\frac{1}{T_{\mathrm{INC}}} E-\frac{R_{\mathrm{QRT}}}{T_{\mathrm{LIC}}} I-\frac{1}{T_{\mathrm{INF}}} I<0 .
$$

Reorganizing the terms in equation (10), one obtains

$$
\frac{1}{T_{\mathrm{INC}}} E<\frac{R_{\mathrm{QRT}}}{T_{\mathrm{LIC}}} I+\frac{1}{T_{\mathrm{INF}}} I,
$$


from which it is possible to observe that $T_{\text {INC }}$ and $T_{\text {INF }}$ are parameters whose values are characteristic of the infection and thus not controllable. In turn, the quarantine rate $R_{\mathrm{QRT}}$ and the time lag between infection and quarantine $T_{\text {LIC }}$ are controllable parameters that depend on the agility and effectiveness of identifying and isolating infected individuals. As one seeks to make the right side of the inequation as large as possible, one shall seek for larger values of $R_{\mathrm{QRT}}$ and smaller values of $T_{\text {LIC }}$. The strategy of quarantine of infected individuals usually works by flattening the epidemic curve. To that end, it is necessary that the number of quarantined infected individuals, $Q$, reach a proportion of the total number of infected individuals, $I$, in the population $N$. The coefficient of quarantine of the infected is defined as

$$
C_{Q}=\frac{Q}{(I+Q)}
$$

for $I+Q>0$, and it measures how much a given set of quarantine parameters $R_{\mathrm{QRT}}, T_{\mathrm{QRT}}$, and $T_{\mathrm{LIC}}$ perform in a given scenario. The coefficient ranges from 0 to 1 that correspond to 0 to $100 \%$ quarantine of infected individuals.

3.2.2. Confinement of Susceptible Individuals. The strategy of confinement of susceptible individuals seeks to remove from circulation a fraction of those who are susceptible to neutralize their exposition to the infection. By decreasing the number of circulating susceptible, $S$, one has the multiplication $R_{t} S I$ assume lower values, such that the rate of change in the number of individuals exposed to the infection decreases accordingly. This strategy can be thought of as relaying a share of the workforce that is susceptible, for example, to a $70-30 \%$ proportion of circulating and confined, respectively.

The rate of change in the confined susceptible is given by

$$
\frac{\mathrm{d} C}{\mathrm{~d} t}=R_{\mathrm{CNF}} S-\frac{1}{T_{\mathrm{CNF}}} C,
$$

in which susceptible individuals enter according to a rate $R_{\mathrm{CNF}}$ and confined individuals leave after being confined for $T_{\mathrm{CNF}}$ days. As a confined individual leaves the compartment, he goes back to the compartment of susceptible individuals since during $T_{\mathrm{CNF}}$, the confined susceptible individuals are isolated from infected individuals. The confinement strategy usually works by flattening the epidemic curve. To that end, it is necessary that the number of confined individuals, $C$, reach a proportion of the total number of susceptible individuals, $S$, in the population $N$.

The coefficient of confinement of susceptible is defined as

$$
C_{C}=\frac{C}{(S+C)}
$$

for $S+C>0$. The coefficient ranges from 0 to 1 that corresponds to 0 to $100 \%$ confinement of susceptible individuals.

3.2.3. Social Distancing. The strategy of social distancing seeks to prevent gatherings, circulation, and exposition to deaccelerate the spread of the epidemic. It includes physical distancing in supermarkets, restaurants, and drugstores, for example, and the use of masks in public places. It is expected that the social distancing will reduce the value of the basic reproduction number, $R_{t}$ by means of restriction of social activities that may induce social interactions, such as circulation of public transport or cultural events, for example. Most commonly, only essential services are allowed to function and nevertheless under considerable changes in regulation. In terms of the equations, the strategy of social distancing works by decreasing the basic reproduction number, $R_{t}$, such that multiplication $R_{t} S I$ will assume lower values and the rate of change in the number of individuals exposed to the infection decreases accordingly, as shown in equation (9).

Lockdowns are considered energic measures due to the amount of changes they bring to the daily routines. Nevertheless, they were shown to be effective to deaccelerate infection rates although they may also cause undesirable economical side effects. Thus, the accurate dimensioning of the duration and severity of social distancing measures and the planning of its time window are fundamental to mitigate its negative effects on the economy while being effective in controlling the surge of infections. The level of what we refer to as social distancing will be calculated by means of the coefficient of social distancing:

$$
C_{S D}=\frac{R_{0}-R_{t}}{R_{0}}
$$

where $R_{0}>0$ is the basic reproduction number, i.e., the basic reproduction number before social distancing.

3.2.4. Vaccination. The strategy of vaccination seeks to transfer individuals directly from the compartment of susceptible to the compartment of removed. It can be observed in the equations of the model that vaccination is the only form of getting immunized without being sick (infected). The equation for the rate of change in the number of susceptible individuals is

$$
\frac{\mathrm{d} S}{\mathrm{~d} t}=-\frac{R_{t}}{T_{\mathrm{INF}}} \frac{S I}{N}-R_{\mathrm{CNF}} S+\frac{1}{T_{\mathrm{CNF}}} C-\frac{R_{\mathrm{VCN}}}{T_{\mathrm{IMN}}} S,
$$

where the last term of the equation represents the outflow of susceptible from the compartment according to a rate of vaccination $R_{\mathrm{VCN}}$ and to a time lag $T_{\mathrm{IMN}}$, which represents the number of days necessary after vaccination until the body produces antibodies enough to fight the disease. When leaving the compartment of susceptible, $S$, the vaccinated individuals enter the compartment of removed according to rate $R_{\mathrm{VCN}} / T_{\mathrm{IMN}}$.

\subsection{Measuring the Combined Effectiveness of Strategies.} In the last section, the presentation of control strategies showed that they operate upon the reduction of the product $R_{t} S I$, one at a time. Note that this term appears as a flow of individuals from the compartment of susceptible to the 
compartment of exposed and then to the compartment of infected, that is, $S \longrightarrow E \longrightarrow I$. When two or more strategies are applied simultaneously, the combined effect of them can be evaluated by means of what we call the effective isolation coefficient, EIC, as

$$
\mathrm{EIC}=1-\frac{R_{t} I S}{R_{0}(S+C)(I+Q)}
$$

where $R_{0}>0$ is the basic reproduction number and $(S+C)>0,(I+Q)>0$; that is, the coefficient is defined for the period during the epidemic. The EIC allows the evaluation of the relation between the severity of the isolation measures and the results upon the infected population. When $R_{0}=R_{t}$ and $Q=C=0, \mathrm{EIC}=0$ and there is no isolation at all, whereas $R_{t}=0$ or $S=0$ or $I=0$ with $R_{0}(S+$ $C)(I+Q)>0$ yielding EIC $=1$, which means that there is no transmission. This coefficient will be applied to the scenarios studied in Section 4. Thus, we reserve the term social isolation to refer to the combined effects of social distancing, confinement of susceptible, and quarantine of infected individuals.

3.4. The Parameters. The clinical parameters used in the model were adopted from previous clinical studies on the assumption that they are roughly the same everywhere. This assumption might be proven mildly inaccurate in the near future, but considering the lack of consolidated data and results at the current moment (April 2020), this seems to be the most viable way to get to a functional and working model for prognostic and strategy planning. Considering several studies in the literature, the parameters of the model are set to the values shown in Table 1 .

The values of $R_{t}, R_{\mathrm{HSP}}, R_{\mathrm{ICU}}$, and $R_{\mathrm{DHT} 1}$ were calculated from the information on confirmed and active COVID-19 cases provided by the Health Secretary of the State of Amazonas [20]. Currently, the amount of data is insufficient to calculate $R_{\mathrm{DHT} 2}$, and thus, it was arbitrarily set to $30 \%$ higher than $R_{\mathrm{DHT} 1}$. The values for $R_{t}\left({ }^{*}\right)$ and $R_{t}\left({ }^{* *}\right)$ resulted from model calibration to the COVID-19 confirmed positive data from Manaus, made available by Health Secretary of the State of Amazonas [20]. Furthermore, the first research with considerable size and scope in Brazil applying widespread testing of individuals chosen at random concluded that subnotification of COVID-19 is considerable, having found that, for every confirmed case, there are at least seven infected individuals EPICOVID19 [27]. We apply this 7-fold factor to compensate the hospitalization rate values $R_{\mathrm{HSP}}$ and $R_{\mathrm{ICU}}$ presented in Table 1. The death rate $R_{\text {DHT1 }}$ is maintained since it is applied upon the rate of critical individuals, $R_{\mathrm{ICU}}$. Since it was not possible to access consolidated data regarding the number of ICU beds available for COVID-19, it was set to $N_{\text {ICU }}=148$, which represents about $30 \%$ of the total number of ICU beds in the State of Amazonas. This is only a reference number and might not reflect the real availability. The number of simple hospital beds for COVID-19 $N_{\text {HSP }}$ was set to 1, 000 for reference under the unavailability of updated information.
3.5. The Model Dashboard. The model dashboard was implemented using Python 3.7 programming language and the Dash Plotly library [28]. The equations are integrated numerically using the fourth-order Runge-Kutta method, with an integration step $\Delta t=1$. The model is presented in the form of an interactive dashboard, and it is available online at https://dashseir.herokuapp.com/. The working of the dashboard is further detailed in the flowchart in Figure 2.

3.6. Model Calibration. Many different groups have delivered relevant research and data concerning the coronavirus outbreak as the epidemic is ongoing and evolving from country to country. It is important to note that official data are hampered by a number of sources of uncertainty: undertesting, lag of test results, and lack of accuracy in infection dating, among others. Furthermore, all research is preliminary in the sense that they deal with rather problematic data sources which are likely to be revised and consolidated in the future. The characteristics of the epidemics and the current stage of promptness of health organs and laboratories worldwide make the infection curve lead the official confirmed cases by a period that might well be of about 10 days or more. Thus, for now, the calibration of epidemic models based on confirmed infection data can lead to highly inaccurate prognostics.

To overcome this limitation, the model was formulated as much as possible on the basis of parameters that can be directly mapped to those of clinical studies. On the contrary, parameters depending on social behavior were calibrated on the basis of available data, such as the reproduction number $R_{t}$. The reproduction numbers for the periods before and during lockdown were calculated by fitting the model to the available official data from the Health Secretary of the State of Amazonas [20]. Calibration processes were performed and were aimed at finding the value of $R_{t}$ that minimizes the root mean square error (RMSE) between the data and the model outputs.

3.7. Case Study. Manaus is the capital of Amazonas state and is located on the banks of Negro river in Northwest Brazil. As the largest city of the state, it occupies an area of $11,401 \mathrm{~km}^{2}$, from which $427 \mathrm{~km}^{2}$ is an urban area, as represented in Figure 3. Manaus currently holds a population of about 2, 15 million people and has recently been pointed as one of the first Brazilian cities that shall face the collapse of health care facilities due to the coronavirus epidemic. Although the actions of the State Government in tackling a possible epidemic have been important to increase the number of available beds for the hospitalization of serious cases and the number of health professionals, the cases of COVID-19 rapidly increased since late March to reach over a thousand cases by April 12th.

Temporary hiring of human resources, purchase of supplies, equipment for laboratory analysis, and personal protective equipment (PPE) are some of the actions that were announced. To expand the supply of health professionals available to deal with the pandemic, the State 
TABle 1: Parameters applied in the study and their sources.

\begin{tabular}{lccc}
\hline Parameter & Value & $95 \%$ CI & Reference \\
\hline$T_{\mathrm{INC}}$ & 5.2 & $4.1-7.0$ & {$[22]$} \\
$T_{\mathrm{HSP}}$ & 12.4 & $7.6-17.2$ & {$[27]$} \\
$T_{\mathrm{ICU}}$ & 8.0 & $4.0-12.0$ & {$[4]$} \\
$R_{t}\left(^{*}\right)$ & 3.55 & - & Our study \\
$R_{t}\left({ }^{*}\right)$ & 1.51 & - & Our study \\
$R_{\mathrm{HSP}}(\%)$ & 13.55 & - & Our study \\
$R_{\mathrm{ICU}}(\%)$ & 8.92 & - & Our study \\
$R_{\mathrm{DHT} 1}(\%)$ & 54.04 & Our study \\
\hline
\end{tabular}

${ }^{*}$ No distancing; ${ }^{* *}$ with social distancing.

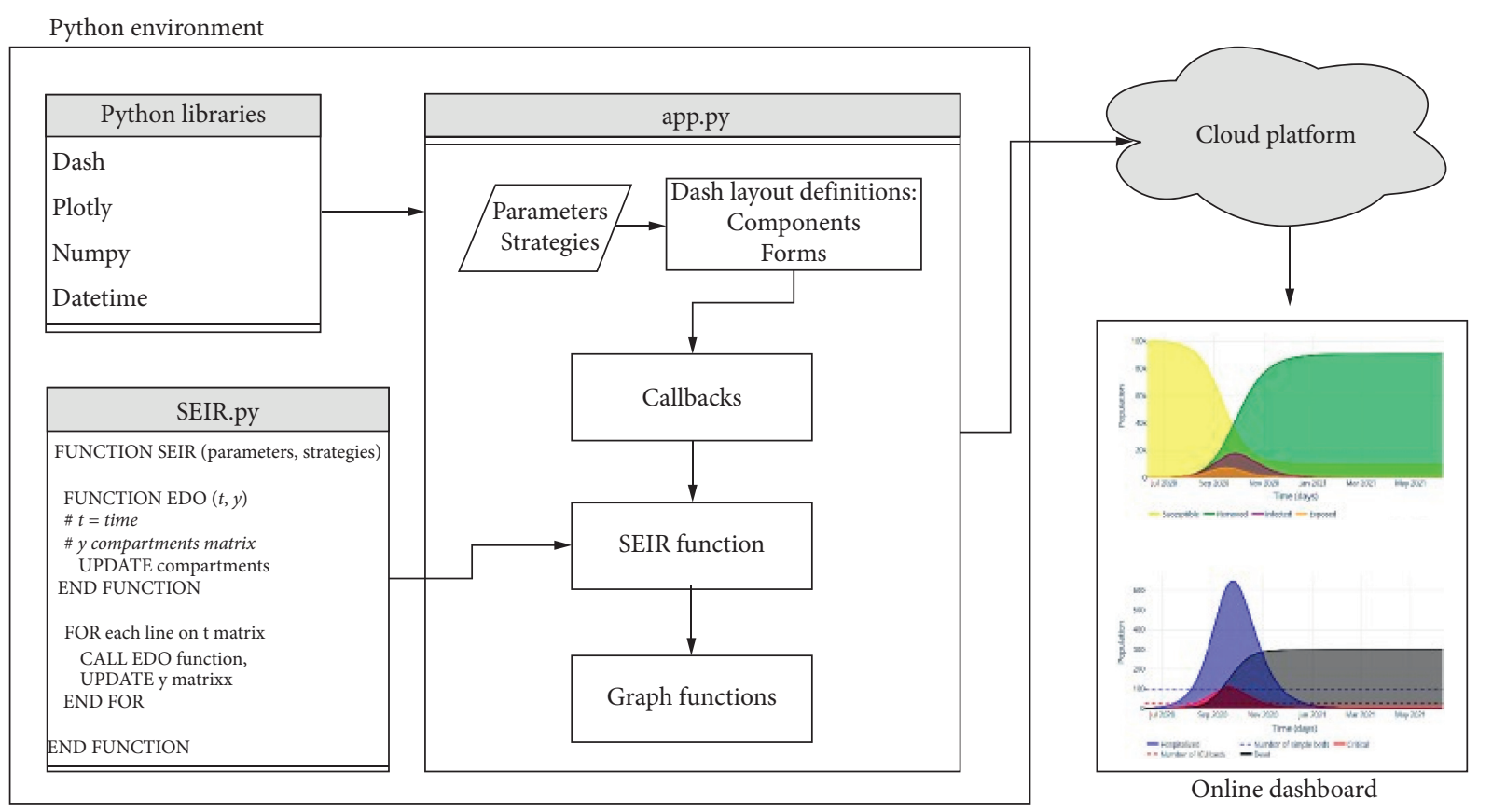

FIgURE 2: Flowchart of the model dashboard.

University of Amazonas (UEA) announced the anticipation of the graduation of 79 doctors, 28 nurses, and 21 pharmacists by six months [20]. The measure aims to optimize the availability of health services and increase the service capacity of public units, especially those receiving suspected and confirmed coronavirus patients.

On March 23rd, the Amazonas state government announced that the state of public calamity, suspended activities from public organs, and oriented novel hygiene and monitoring procedures in industries. However, the rapid growth in the number of cases since then until the first days of April prompted the state to take more forceful measures. On April 4th, the state government announced further restrictive measures to contain the epidemics: schools were closed and classes broadcasted on open TV channels; only essential services remained working; and the transportation of passengers in buses, taxis, and vans was suspended in federal and state roads. Since then, the number of cases and deaths kept growing rapidly and the capital Manaus became a major concern due to the concentration of confirmed cases and deaths of coronavirus patients.
According to official data, from the current 863 active cases, 117 are hospitalized in mild conditions and 77 are critical and occupying an ICU bed. Furthermore, there are currently another 366 hospitalization of cases under investigation, 313 in regular beds and 53 in ICU beds [20]. The evolution of the epidemic in Manaus (AM) is presented in Figure 4.

From the official and confirmed active cases, one can calculate the percentages of hospitalization in regular and ICU beds to be $13.6 \%$ and $8.9 \%$, respectively. Although these are preliminary data, it is the major source of information in the current situation, and thus, these parameters will be applied in the model to evaluate the deficit in medical infrastructure in each scenario.

\section{Model Application}

The expanded SEIRCQ model was run on the data from Manaus in order allow the calibration of parameter $R_{t}$ that depends on social behavior. The number of ICU beds used in this study corresponds to those informed in publications by 

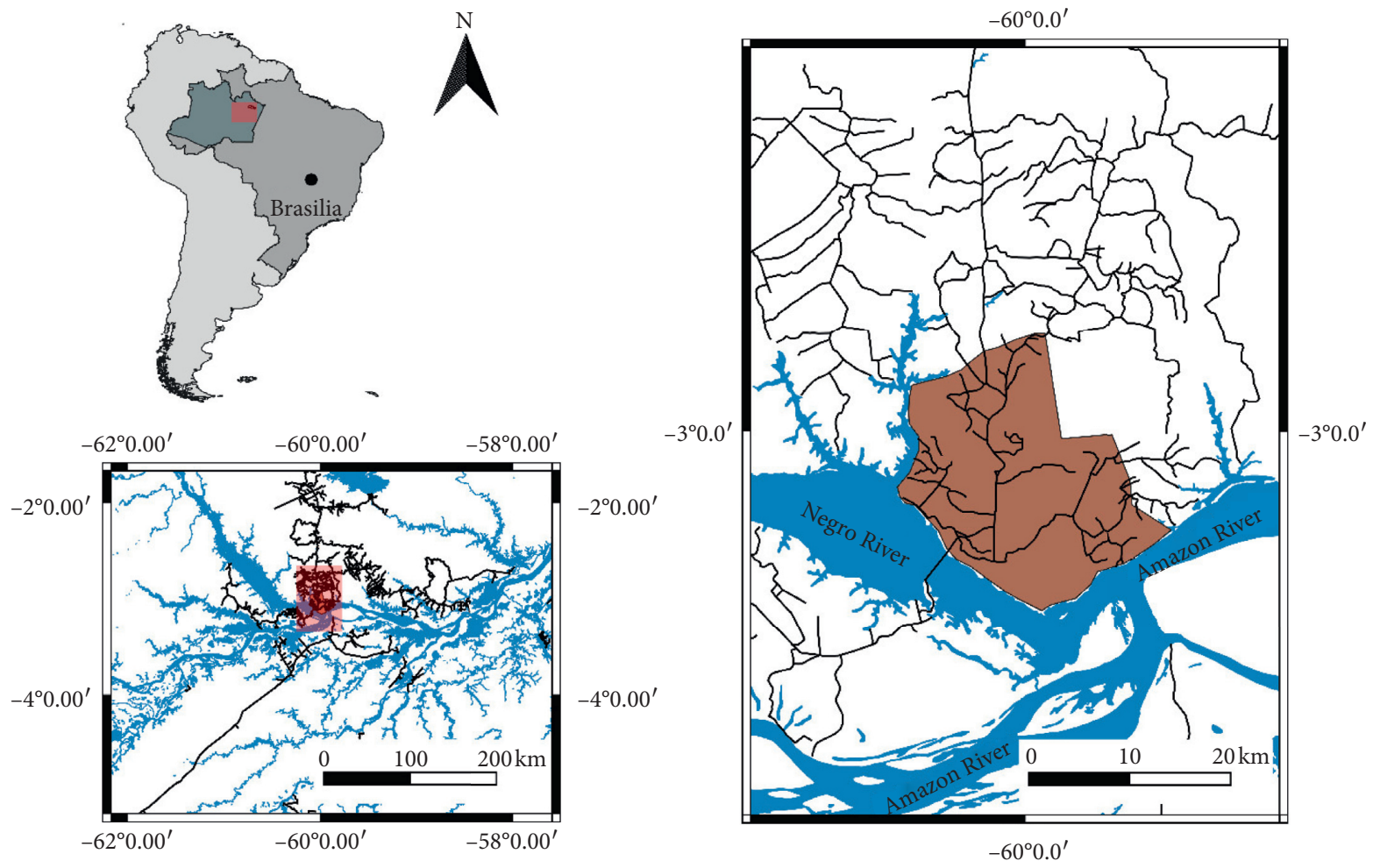

South America

Brazil

Amazonas state

Manaus city

Main roads

Rivers and lakes

Figure 3: Manaus, the capital of the State of Amazonas, Brazil.



Figure 4: Number of confirmed cases and deaths due to COVID19 in Manaus (AM), Brazil.

official organs [20]. New ICU beds are not taken into consideration since they are not available yet, and there is not clarity about how many extra beds will be made available and when. Furthermore, the number of ICU beds indicated as $N_{\text {ICU }}$ in the model refers to vacancies for COVID-19 patients.

4.1. Model Calibration for Manaus, Brazil. The curves showing the number of confirmed cases (real vs. model) are shown in Figure 5. These curves correspond to the initial phase of the epidemics, before the first governmental restriction decree took effect. Recall that there is a delay

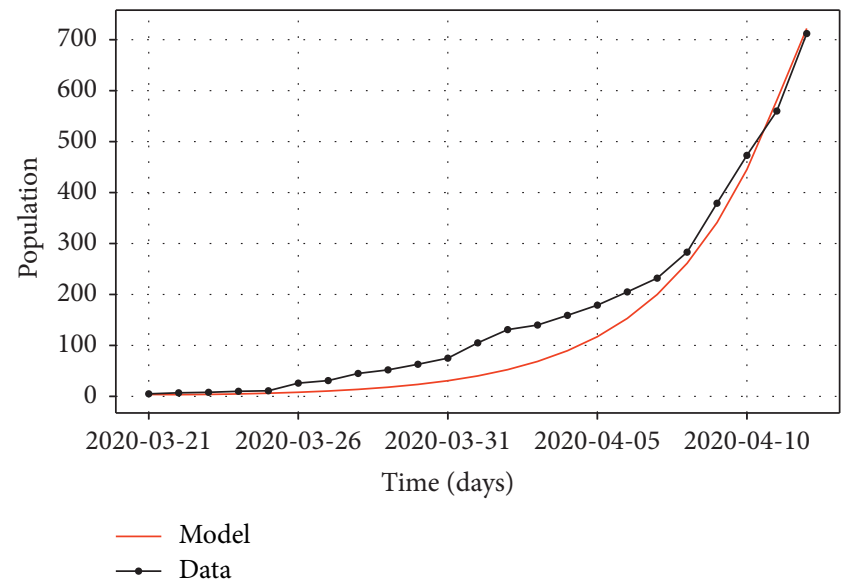

Figure 5: Model calibration for the data from Manaus, Brazil, before isolation measures: the basic reproduction number was found as $R_{0}=3.55$ and thus larger than most values found in the literature $[2,7,22]$.

between the exposition and symptoms (if any) and another delay between symptoms and testing/confirmation by official organs. The time lag between the first social distancing measures and their visible effects in the epidemic curve was of about 14 days.

The epidemic growth already shows a slowly decreasing growth rate. Model calibration for the available data of the 
first social distancing period resulted in reproduction number $R_{t}=1.51$. The calibration curves are shown in Figure 6.

As plotted in a single graphic window, the model and the data curves present a sigmoidal shape, which may indicate that the epidemic is reaching a plateau. Is that so? The next section shows that if current social distancing measures are maintained to the same levels, the number of confirmed cases would grow steadily for weeks before starting to decelerate.

The calibration showed that the infection curve started to decelerate in the beginning of April, about 14 days after the isolation measures became effective. As expected, there is a time lag between the implementation of isolation measures and its visible effects in the infection curve. This should also be taken into account in planning epidemic control measures. As the model results described the infection curves, all the dates mentioned will be referred to the official confirmed data, which means that they will be presumably delayed relatively to the real infection curve.

4.2. Exploring the Application Epidemic Control Measures: Alternative Scenarios and Actions. Next, we present three case studies that consider different scenarios for 180 days following the first efforts of social isolation which occurred in March 2020. Three scenarios are considered as prognostics of the epidemic:

(i) Scenario 1 applies the current level of social distancing (EIC of about 0.57).

(ii) Scenario 2 applies more severe social distancing measures (EIC of about 0.70).

(iii) Scenario 3 applies more severe social distancing measures (EIC of about 0.80 ).

4.2.1. Scenario 1: Epidemic Prognostics under Mild Social Distancing Measures (as Currently Adopted). The following simulation presents a prognostic of the likely scenario under $\mathrm{EIC}=0.57$, which roughly corresponds to the calibrated parameters that reflect the current situation by April 2020. Although the current measures helped reduce the rate of infection as compared to the initial stages of the epidemic, the model shows that the sigmoid-shaped curve would not lead to a plateau in the following days, as one might believe by checking Figure 7. Rather, the number of infected people shows a tendency to accelerate in the following weeks.

The hospitalization curves for this scenario are shown in Figure 8. Note that, under this scenario, the number of ICU beds would be exceeded in late April and the number of patients continue to rise for about two months. Under this scenario, the epidemic would reach a peak only in the period from mid-June to early July and drag the health care system into a period of overload, in which case the available medical infrastructure would be greatly exceeded. Indeed, Manaus and more broadly the Amazonas state are known to have a low average number of ICU beds of about 1.24 to each 10.000 inhabitants. The problem would be worsened by the

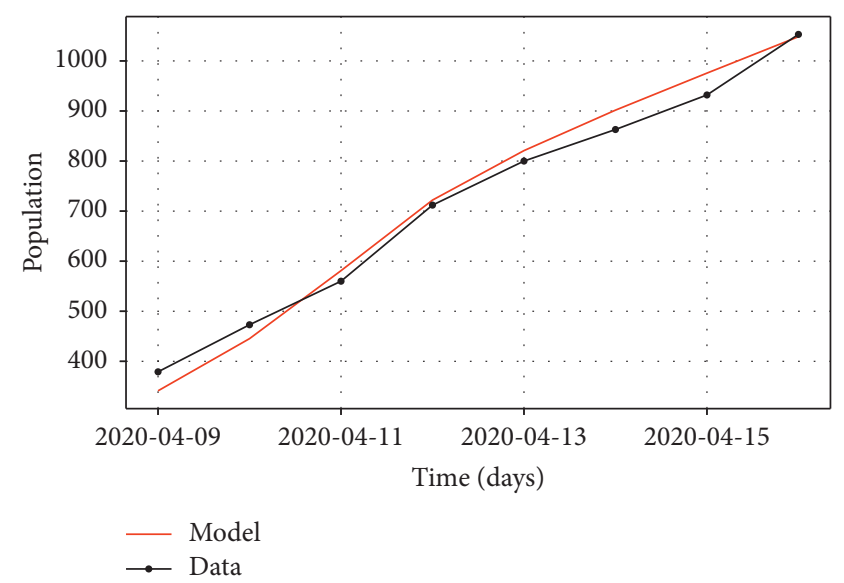

Figure 6: Model calibration for the data from Manaus (Brazil) after isolation measures: the reproduction number was found as $R_{t}=1.51$.

insufficient reduction of the basic reproduction number under distancing, which is an effect of social behavior.

For the same scenario, Figures 9 and 10 show the peak of the epidemics expected to happen in mid-June. If hospitalization rates continue at the same levels, the hospitalizations would amount to a couple thousands at the peak, as shown in Figure 10. Note that, under this scenario, the medical infrastructure would be greatly and rapidly exceeded beyond any feasible perspective of building new ICU beds. Figure 11 shows the effective isolation coefficient for scenario 1.

Of course, as the perspective of such a scenario becomes a reality, more strict measures are likely to be taken and social behavior naturally would change. How about alternative scenarios of more severe social distancing measures? How would they impact the overload in the medical infrastructure? This is a question on which the next section attempts to shed some light.

4.2.2. Scenario 2: More Strict Social Distancing Measures. By making the distancing measures more severe, the epidemic curve would reach a maximum and then start to decline by the end of May. At this time (April 2020), Manaus is facing the beginning of the exhaustion of the medical infrastructure. In a scenario of more intense social distancing measures in which the reproduction number would decrease to $R_{t}=1.10$, the curve would reach a peak and then slowly start to decline. As such measures last for about 90 days, they would support a consistent reduction in the number of infected people. Figure 12 shows the prognostics of the numbers of deaths under this scenario, and Figure 13 focuses on hospitalizations.

It is noticeable that even though the epidemic is controlled under $0.70 \mathrm{EIC}$, the number of ICU beds would be insufficient. Under this scenario, the number of critical patients would reach nearly double of available ICU beds. It is important to note that Manaus concentrates the ICU beds in the State of Amazonas, and thus, the total count of ICU beds required statewide would be considerably higher. 


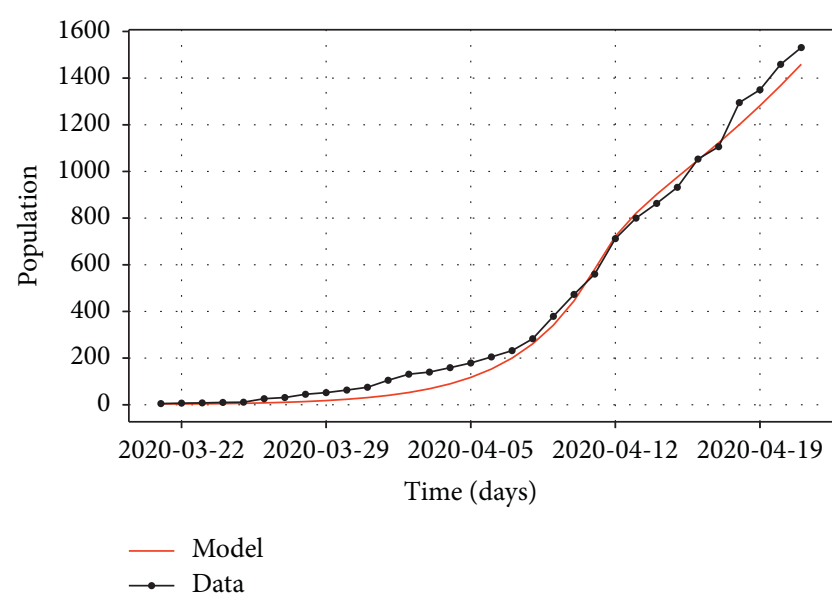

FIgURE 7: Sigmoid-shaped curves of the model and data for Manaus, Brazil.

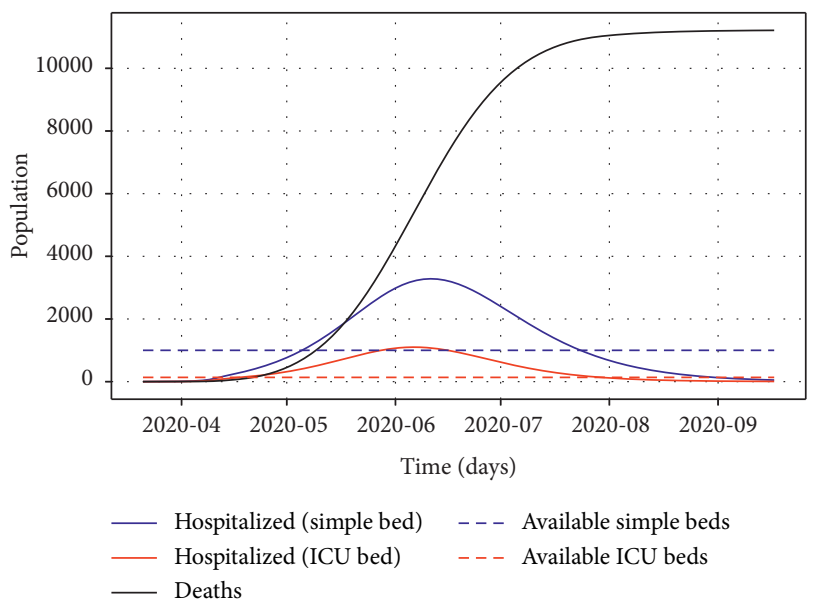

Figure 8: Prognostics based on the model calibration for Manaus, Brazil: scenario for 180 under current distancing measures of social distancing.

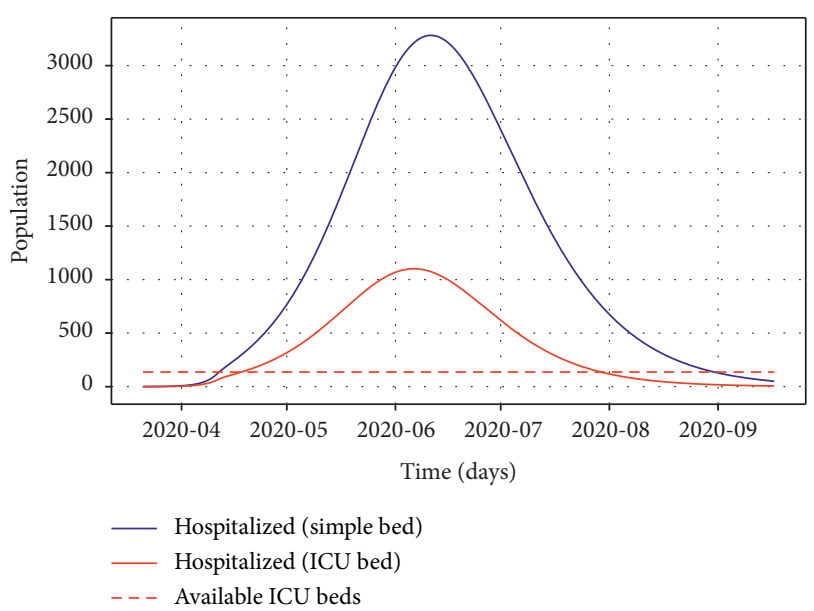

Figure 9: Prognostics based on the model calibration for Manaus, Brazil: scenario for 180 days under the current distancing measures.

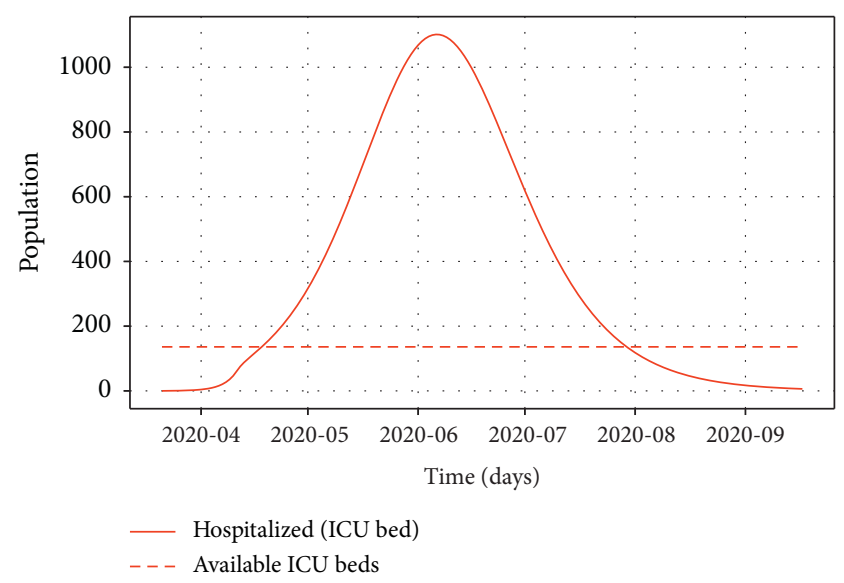

Figure 10: Prognostics of hospitalizations in simple beds and ICU beds for 180 days under the current distancing measures.



FIGURE 11: Effective distancing coefficient for the first scenario (mild social distancing), as currently applied.

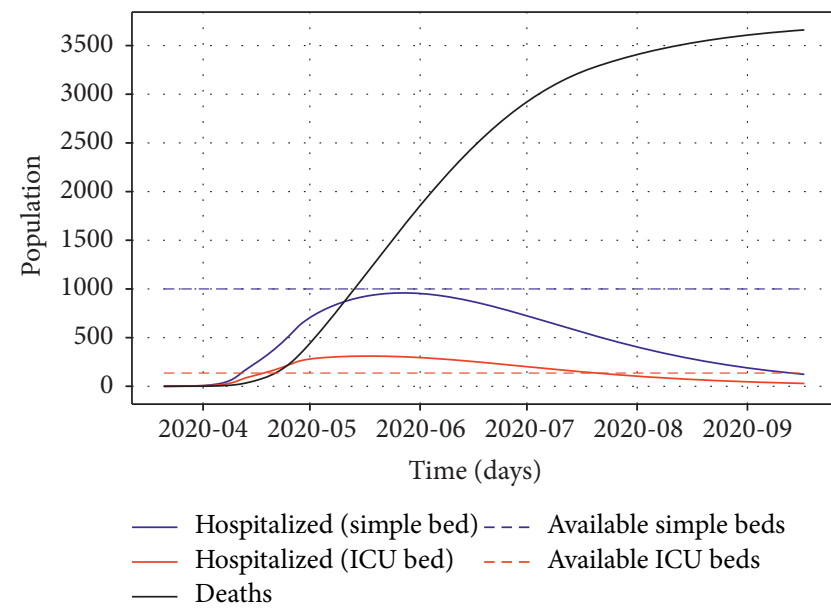

FIgURE 12: Prognostics of deaths and hospitalizations in simple beds and ICU beds for 180 days with $R_{t}=1.51$ and then $R_{t}=1.10$. 


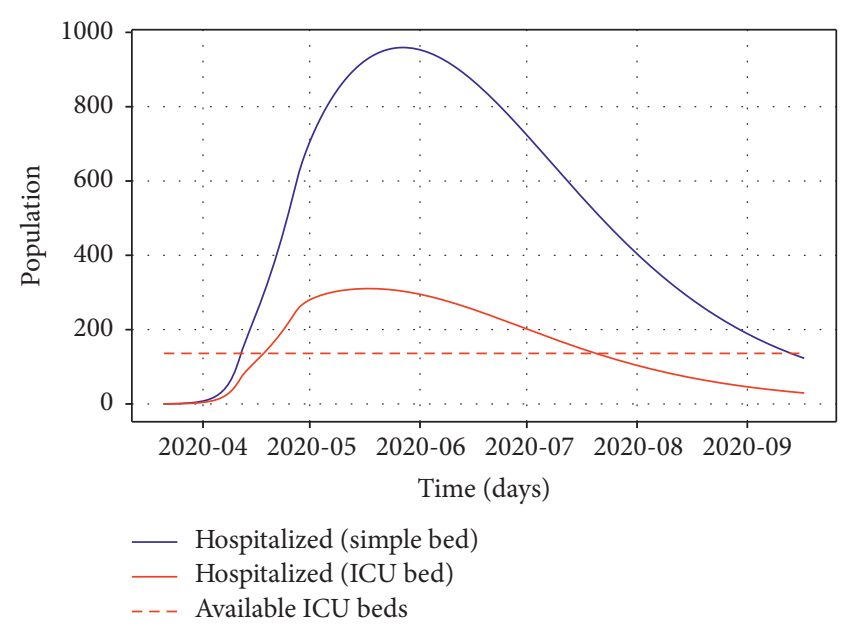

FIgURE 13: Prognostics of hospitalizations in simple beds and ICU beds for the following 180 days with $R_{t}=1.51$ and then $R_{t}=1.10$.

Moreover, under this scenario, about 3 months ahead of April, the number of required ICU beds would be nearly the total capacity, as shown in Figure 14. It should be noted that the model does not take into account that equipment may become unavailable as they break, as well as medical staff might themselves be unavailable as they might be infected during the epidemic. It is noticeable that even under more strict distancing measures, the existing infrastructure would be insufficient. Figure 15 shows the EIC for scenario 2 .

Under this scenario, a consistent decline in the number of infected is observed and the number of infected individuals would return to the current level after a steep ascent in the last week of April. However, strict social distancing measures might be hard to implement in practice since there are economic issues that have to be taken into account. There are other alternatives to help handle this situation. The next section gives a hybrid approach in which three epidemic control strategies are simultaneously applied to reduce infection rates while also maintaining the economy running as much as possible.

4.2.3. Scenario 3: Severe Social Distancing and Confinement, Followed by Quarantine of Infected Individuals. This scenario considers an EIC of about 0.80 during four to six weeks, such that the spread of the virus is further reduced relatively to the previous scenarios. In this scenario, the reproduction number is decreased to $R_{t}=1.10$ by more severe social distancing measures. This period would ideally start as soon as possible (the simulations considered late April) and last for about 45 days. After that, the more strict social distancing measures could be relaxed to levels comparable to those imposed by the government decree from March 23 but added with two other strategies: confinement (of susceptible) and quarantine (of infected). The confinement of the susceptible is at the rate of $2 \%$ per day, that is, $R_{\mathrm{CNF}}=0.02$ and $T_{\mathrm{CNF}}=15$, which would result in a peak of about $20 \%$ of the total susceptible population being confined. About four weeks later, as the

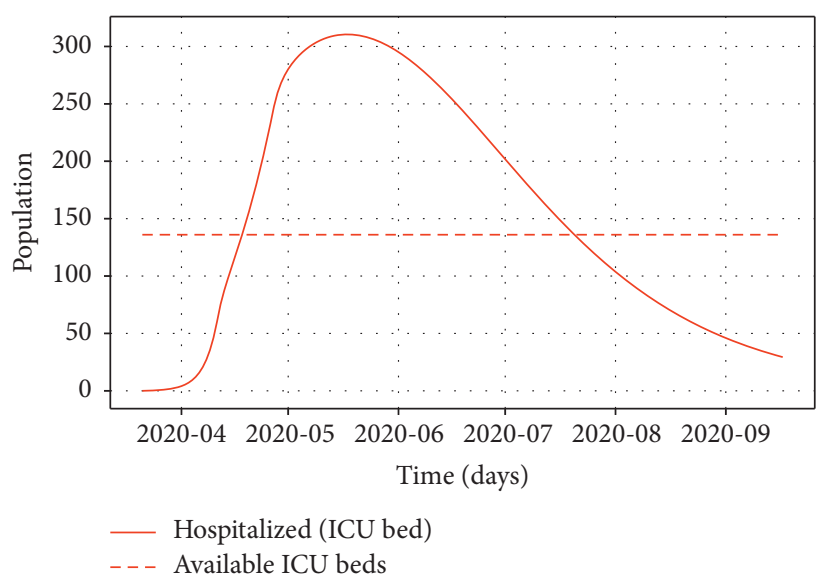

FIGURe 14: Prognostics of hospitalizations in ICU beds for 180 days with $R_{t}=1.51$ and then $R_{t}=1.10$.

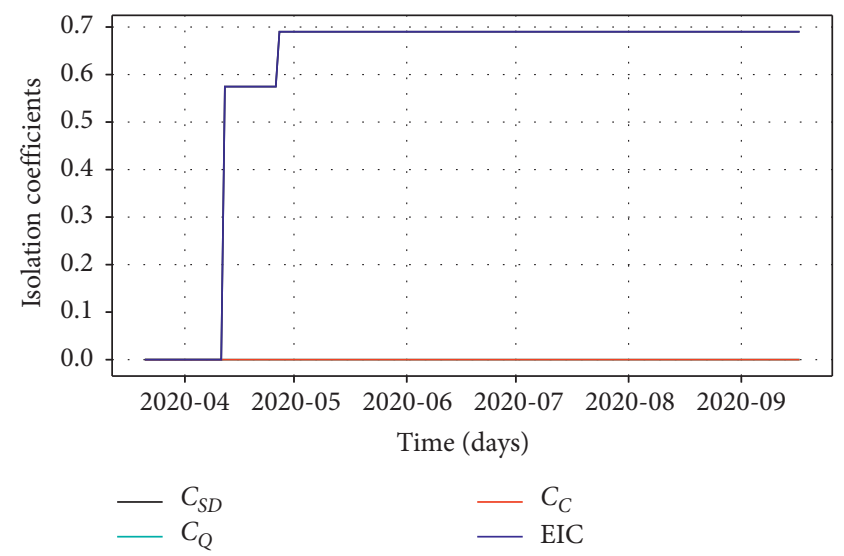

FIGURE 15: Effective distancing coefficient for the second scenario, which would stabilize the epidemic (interrupting mild distancing and implementing severe distancing).

more severe social distancing period reaches about $2 / 3$ of its duration, the quarantine of infected individuals would take place with $R_{\mathrm{QRT}}=0.10, T_{\mathrm{QRT}}=15$ days, and $T_{\mathrm{LIC}}=2$ days. It shall be remarked that the quarantine of infected individuals heavily relies on testing, clinical diagnostics, and adequate social behavior. The period of more severe social distancing measures would give authorities time to put into practice testing campaigns starting in late May or early June.

Under such conditions, the epidemic would be maintained under control after a peak in mid-May, as shown in Figures 16 and 17. As a result of controlling the epidemic, the curve of hospitalized individuals is found to behave satisfactorily since it remains below the presumable number of available beds. In this case, the peak would overwhelm the medical infrastructure during a short period after which the number of hospitalizations in ICU beds would consistently decrease, as shown in Figure 18. The EIC for scenario 3 is shown in Figure 19.

This strategy would require that widespread testing be performed upon mid-June 2020 upon the mild relaxation of social distancing measures. 


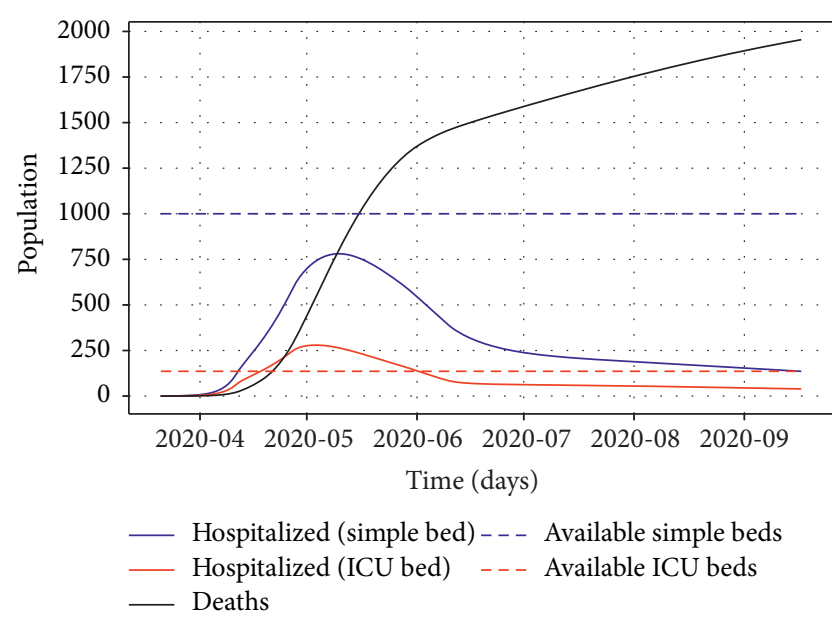

Figure 16: Prognostics of death under mild and severe isolation measures followed by simultaneous application of confinement of susceptible $\left(R_{\mathrm{CNF}}=0.02\right.$ and $\left.T_{\mathrm{CNF}}=15\right)$ and quarantine of infected $\left(R_{\mathrm{QRT}}=0.10, T_{\mathrm{QRT}}=15\right.$, and $\left.T_{\mathrm{LIC}}=2\right)$ individuals.

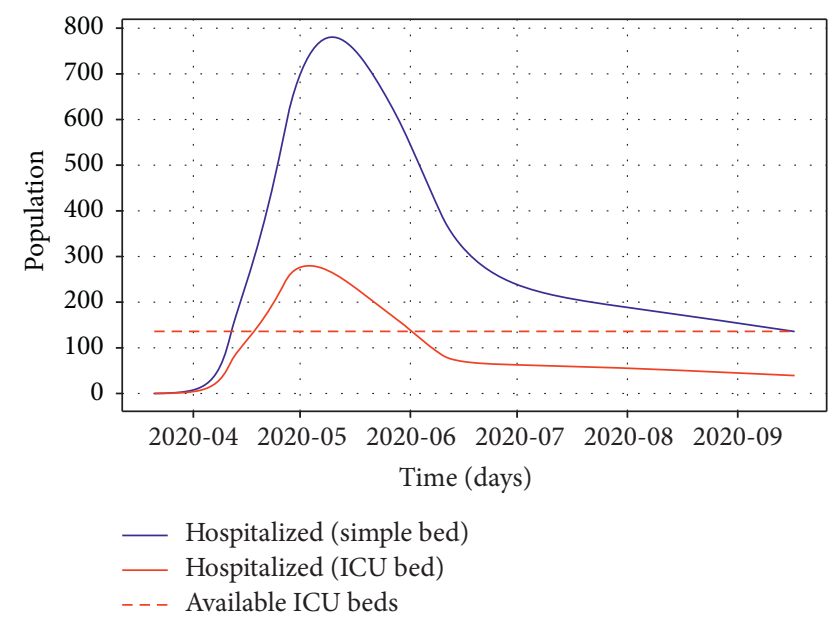

FIgURe 17: Prognostics of hospitalizations under mild and severe isolation measures followed by simultaneous application of confinement of susceptible $\left(R_{\mathrm{CNF}}=0.02\right.$ and $\left.T_{\mathrm{CNF}}=15\right)$ and quarantine of infected $\left(R_{\mathrm{QRT}}=0.10, T_{\mathrm{QRT}}=15\right.$, and $\left.T_{\mathrm{LIC}}=2\right)$ individuals.

\section{Discussion}

5.1. The Importance of Mathematical Models in This Epidemic. As the number of cases of COVID-19 in the world surpasses a couple millions, the effects of exponential growth seem to continue misunderstood. This is quite problematic since most of the success or failure of strategic control measures relies upon how they are implemented in practice by society as a whole. In this sense, models can have a widespread didactic effect upon the individual comprehension of the dynamics of the epidemic and how fast things can deteriorate.

As the world goes through one of the most challenging periods in recent history, the application of all available knowledge that can gain us time is needed to deal with the limitations of health care systems and search for effective

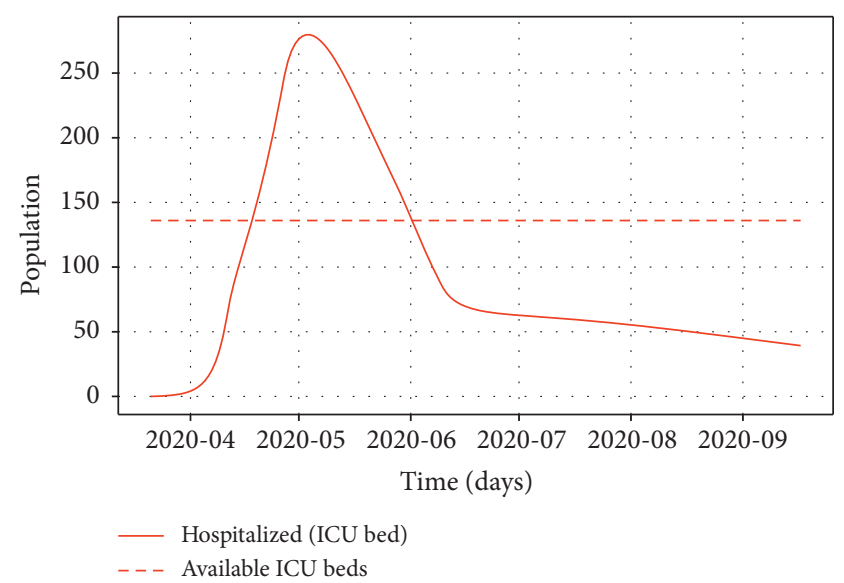

FIgURe 18: Prognostics of hospitalizations in ICU beds under mild and severe isolation measures followed by simultaneous application of confinement of susceptible $\left(R_{\mathrm{CNF}}=0.02\right.$ and $\left.T_{\mathrm{CNF}}=15\right)$ and quarantine of infected $\left(R_{\mathrm{QRT}}=0.10, T_{\mathrm{QRT}}=15\right.$, and $\left.T_{\mathrm{LIC}}=2\right)$ individuals. The cumulative effective of the three strategies combined presents an IEC of about 0.80 .

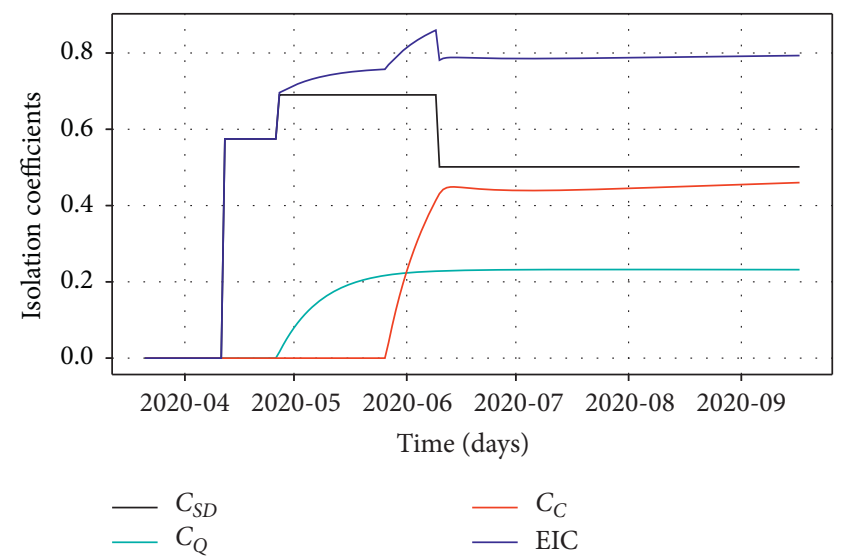

FIgURE 19: Cumulative effect of the isolation measures. Effective isolation coefficient for the third scenario with three control strategies in use, which would stabilize the epidemic faster and allow greater retake of economical activity (interrupting mild distancing and implementing severe distancing along with confinement of susceptible and then effective quarantine of infected).

treatments that can help reduce the death toll. In this context, gaining time is mainly a matter of strategically dealing with this first wave of the epidemic. To this end, scientific, medical, and political personnel are working to flatten the curve as much as possible and make it fit into the health care system capacity. Naturally, not the same strategy will work everywhere. Meanwhile, there is enormous concern about the impacts of the epidemic and of the control strategies in the economy, as well as about a second wave of the epidemic. As such, humanity is currently living in an ongoing multiobjective optimization problem whose outcome greatly depends on early action.

In this sense, the application of mathematical models can be worth an alternative to gain time to find solutions that can be effective to simultaneously preserve public health and mitigate the deep effects that the epidemic shall have upon 
production, employment, and the economy as a whole. While the obvious best solution from the point of view epidemic control is a complete lockdown for about 4 to 6 weeks, this is not economically affordable nor physically feasible for many cities, states, or countries without severe collateral damage, such as the consequences to economically vulnerable people. As such, alternative strategies must be considered in such cases, and they should be tested and perfected in mathematical models before application.

\subsection{From the Mathematical Model to the Real Epidemic.} Regarding the results of mathematical models and their application in practice, some matters of utmost importance are (i) how to translate and relate the theoretical control actions and parameters into practical control parameters of the real-world epidemic and (ii) how to evaluate the evolution of the control measures in the real-world epidemic? While the results of control strategies from the models seem promising, they are of no practical interest if we fail to map them to real life. To illustrate how this can be done, let us consider one strategy at a time.

5.2.1. Social Distancing. Social distancing was shown to have effect in the reproduction number, $R_{t}$, since the parameter represents the interaction between individuals that will result in exposition to the infection. This study found the basic reproduction number as $R_{0}=3.55$ for Manaus and then $R_{t}=1.51$ after the government decree of March 23rd, imposing a rather mild set of social distancing measures. Social distancing in Brazil has been measured by means of smartphone apps, and for the period under study, the State of Amazonas achieved indices of reduction of mobility of the order of $50 \%$, which reflected in the reduction of the reproduction number in the period. Thus, although monitoring methodologies may find enhancements in the following weeks, they seem to be sufficiently adequate to allow the verification of the control parameter $R_{t}$ in practice.

While other methodologies might be available, such image processing from aerial images by vants or street surveillance cameras, the individual localization of a fraction of the population by means of smartphone apps seems to give a sharper account of the mobility dynamics of a given geographic area. To this end, the mapping of EIC to isolation indices from smartphone monitoring can help provide a link between the model parameter and the real-world epidemic.

5.2.2. Confinement of Susceptible Individuals. The simulations for scenario 3 applied the confinement rate $R_{\mathrm{CNF}}=$ 0.02 (or $2 \%$ of individuals per day) and confinement period $T_{\mathrm{CNF}}=15$ days. While the confinement period is rather intuitive and easy to understand, the confinement rate might be a bit trickier, especially when it comes to translating it into clear instructions on how to proceed in practice. For a big company, it could represent the entering of $2 \%$ of its (supposedly) susceptible employees in isolation in a daily basis and leaving isolation after $T_{\mathrm{CNF}}$ days. This would create a flow of susceptible to confined and back to susceptible that for these parameter values would reach a peak of $20 \%$ of the employees confined simultaneously. Although this can be clear enough as an instruction to employees in a big company, it would be rather confusing and hardly feasible to society as a whole. Thus, a different approach would be to confine individuals in the proportion of the peak of the curve. In the example of Manaus, where about 400,000 would be confined in the peak, this would represent roughly $20 \%$ of the population.

Thus, as a rule of thumb, after checking the strategy in the model, the government could suggest the confinement of $20 \%$ of (supposedly) susceptible individuals to be implemented everywhere except basic services. The results of such measure could also be assessed using the monitoring of the location of smartphone devices. It is important to note that this strategy works best upon testing to assure that the individuals being confined are effectively susceptible ones.

5.2.3. Quarantine of Infected Individuals. The simulations in Section 4.2.3 applied quarantine rate $R_{\mathrm{QRT}}=0.10$ (or $10 \%$ of the infected individuals that were identified during the period $T_{\text {LIC }}$ ), quarantine period $T_{\mathrm{QRT}}=15$ days, and average delay until quarantine of an infected individual as $T_{\text {LIC }}=2$ days. While the confinement strategy deals with individuals that cannot spread the infection by themselves, the quarantine deals with more hazardous elements of the population that should be monitored more closely. As tests are lacking in Brazil and the ones applied take long until any conclusive result is achieved, this strategy has been applied on the basis of clinical exam of symptomatic individuals. Thus, under the current conditions and considering recent studies that found that, for each COVID-19 confirmation, there are about at least 7 asymptomatic or mild positives that were missed, it would be very far from reality to set the quarantine rate to much larger values than $10 \%$. Quarantined infected must be monitored by health agencies during $T_{\mathrm{CNF}}$ to guarantee that they comply with the quarantine period under the right conditions to make it effective In practice and with time, this strategy would be equivalent to effectively quarantine about $70 \%$ of the infected individuals at the peak since the strategy would greatly reduce the inflow of newly exposed individuals. This requires intensive testing even in asymptomatic individuals, and for this reason, it was left for application from early June such that authorities have time to prepare massive testing campaigns.

As shown in the simulations for the case of Manaus, having this strategy taken into effect would allow that the number of infections progressively decreases along with the number of quarantined individuals after about a month. Under these parameters, the number of quarantined and the number of infected in circulation become roughly the same with time and decrease together to reach values that would clear the overload of the medical infrastructure by the midJune, 2020. 


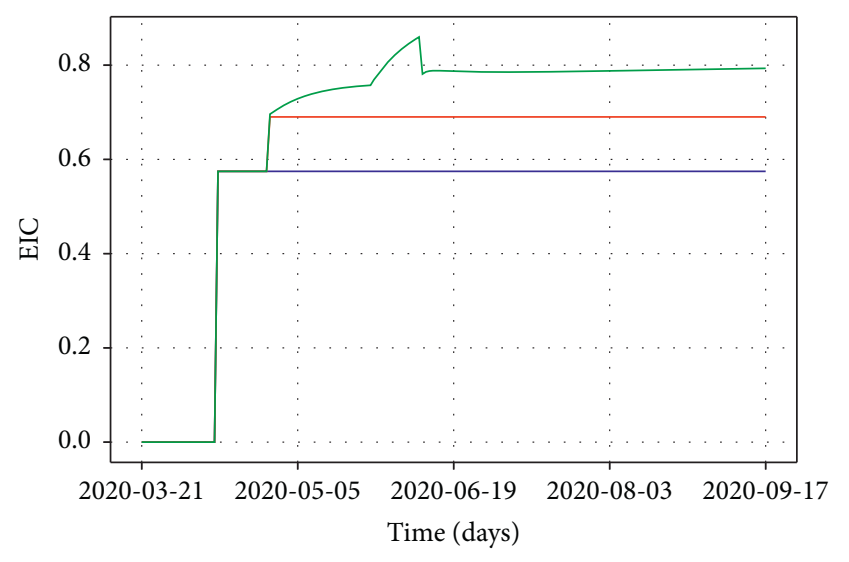

Scenario 1
- Scenario 2
- Scenario 3

FIGURE 20: Effective isolation coefficient for each of the scenarios presented as case studies.

5.3. The Model Results and Infrastructure Planning. The results indicate that the existing medical infrastructure shall be overwhelmed soon under all scenarios considered in the study. How overwhelmed will fundamentally depend on the course of actions and measures from the last weeks of April on.

The first scenario considers the continuity of the current distancing measures reaching an EIC of about 0.57 and, in this case, the number of infected individuals skyrockets. In this case, the number of required simple beds and ICU beds would go well beyond the possibility of the current medical infrastructure and possibly any new infrastructure that might be added in the following weeks.

The second scenario considers intensifying the current distancing measures such that the EIC reaches about 0.70 and the maintenance of this scenario for at least ten to twelve weeks. In this case, further maintenance of distancing measures would cause a consistent decrease in the number of infected and hospitalized and bring the number of infections under control a few months later. In this scenario, the number of ICU beds would be overwhelmed by about $100 \%$ of capacity as well in the peak of this first wave of infection. Although this is well over the current capacity, newly installed ICU beds can be achieved timely if isolation measures are taken soon and the medical infrastructure is expanded accordingly.

The third scenario considers intensifying the current distancing measures such that EIC reaches 0.80 with the confinement of susceptible and quarantine of infected individuals for four to six weeks, along with confinement of susceptible that would reach $20 \%$ of confined at peak. After 30 days, the policy of isolating and monitoring infected individuals would be established, and after 45 days, the measures could be relaxed to the levels observed in early April. Under this scenario, ICU beds would be overwhelmed during a much shorter period. This is a deficit more easily reachable by building new infrastructure than the previous ones.
5.4. The Effect of Isolation. From the EIC values obtained in each scenario, the effect of isolation upon the epidemic curves became clear. Compared to one another, the strategy presented in scenario 3 shows far more effectiveness than the other two, yet it allows further relaxation of isolation in the period following the period of severe isolation. This can give some insight into the role of severe social distancing measures to allow a more rapid resume of economical activities. The EIC for the three scenarios is presented in Figure 20.

\section{Concluding Remarks}

The scenarios presented in this study show that the outcomes are highly dependent upon both the strength and timing plus duration of application of epidemic control strategies. As an unprecedented epidemic in recent history and the first worldwide epidemic of large scope to hit the globalized and interconnected world, the coronavirus outbreak is a novel experience and, as such, has demanded the mobilization of efforts from the most varied fields. As some countries and cities are especially prone to the occurrence of major human disasters in the midst of this pandemic, it is urgent that control strategies are meticulously studied and be ever changed as necessary in order to attend their function of controlling the epidemic, minimize deaths, and minimize economic loss which leads to poverty and misery.

The case of Manaus is very emblematic since the epidemic is at the beginning (as this manuscript is written in mid-April 2020), and there are forms of avoiding more severe consequences if actions are taken timely and with the adequate severity that the current situation calls for. Many other possible measures and strategies can be tested using the same set of parameters or new ones that eventually become available in the next days in the medical literature. Nevertheless, the current state-of-affairs indicates that the medical infrastructure shall be overwhelmed for at least four weeks regardless of any actions that will be taken to contain the epidemic at the moment. This further illustrates the importance of timely and adequately strong isolation measures. This is especially relevant due to the fact that the epidemic curve is currently leading isolation measures by about two weeks, which means that any actions taken now will reflect on the curves in about two weeks.

This study aimed at presenting a model with simple application for the study of COVID-19. We presented the SEIRCQ model and its dashboard developed and implemented in Python language. The dashboard allows the reproduction of the studies presented in this research and several others, and it is available online free of charge at https://dashseir.herokuapp.com/. We encourage the application of the dashboard by health system authorities for applied studies such that they can draw their own possible scenarios when dealing with strategies to combat the epidemic. Furthermore, a case study is presented of the first wave of the epidemic in Manaus (AM), Brazil, with prognostics for a time window of about 150 days under three hypothetic scenarios. 


\section{Data Availability}

The data used to support the findings of this study are available from the corresponding author upon request.

\section{Conflicts of Interest}

The authors declare that they have no conflicts of interest.

\section{Acknowledgments}

The authors thank the Epidemiological Surveillance Service and Committee on COVID-19 Response from the municipality of Erechim, Brazil, for feedback on the model and dashboard.

\section{References}

[1] Q. Bi, Y. Wu, S. Mei et al., "Epidemiology and transmission of COVID-19 in Shenzhen, China: analysis of 391 cases and 1,286 of their close contacts," The Lancet Infectious Diseases, 2020.

[2] D. Wang, B. Hu, C. Hu et al., "Clinical characteristics of 138 hospitalized patients with 2019 novel coronavirus-infected pneumonia in Wuhan, China," JAMA, vol. 323, no. 11, pp. 1061-1069, 2020.

[3] H. Wang, Z. Wang, Y. Dong et al., "Epidemiological and clinical characteristics of 99 cases of 2019 novel coronavirus pneumonia in Wuhan, China-a descriptive study," Lancer, vol. 395, pp. 507-513, 2020.

[4] F. Zhou, T. Yu, R. Du et al., "Clinical course and risk factors for mortality of adult inpatients with COVID-19 in Wuhan, China: a retrospective cohort study," The Lancet, vol. 395, no. 10229, pp. 1054-1062, 2020.

[5] E. J. Emanuel, G. Persad, R. Upshur et al., "Fair allocation of scarce medical resources in the time of covid-19," New England Journal of Medicine, vol. 382, no. 21, pp. 2049-2055, 2020.

[6] J. Michie, "The covid-19 crisis-and the future of the economy and economics," International Review of Applied Economics, vol. 34, no. 3, pp. 301-303, 2020.

[7] T. Kuniya, "Prediction of the epidemic peak of coronavirus disease in Japan, 2020," Journal of Clinical Medicine, vol. 9, no. 3, p. 789, 2020.

[8] Q. Lin, S. Zhao, D. Gao et al., "A conceptual model for the coronavirus disease 2019 (COVID-19) outbreak in Wuhan, China with individual reaction and governmental action," International Journal of Infectious Diseases, vol. 93, pp. 211216, 2020.

[9] W. C. Roda, M. B. Varughese, D. Han, and M. Y. Li, "Why is it difficult to accurately predict the COVID-19 epidemic?" Infectious Disease Modelling, vol. 5, pp. 271-281, 2020.

[10] W. O. Kermack and A. G. McKendrick, "Contributions to the mathematical theory of epidemics-I," Bulletin of Mathematical Biology, vol. 53, no. 1-2, pp. 33-55, 1991.

[11] W. O. Kermack and A. G. McKendrick, "Contributions to the mathematical theory of epidemics-II. The problem of endemicity," Bulletin of Mathematical Biology, vol. 53, no. 1-2, pp. 57-87, 1991.

[12] W. O. Kermack and A. G. McKendrick, "Contributions to the mathematical theory of epidemics-III. Further studies of the problem of endemicity," Bulletin of Mathematical Biology, vol. 53, no. 1-2, pp. 89-118, 1991.
[13] H. W. Hethcote, "The mathematics of infectious diseases," SIAM Review, vol. 42, no. 4, pp. 599-653, 2000.

[14] C. Vargas-De-León, "On the global stability of SIS, SIR and SIRS epidemic models with standard incidence," Chaos, Solitons \& Fractals, vol. 44, no. 12, pp. 1106-1110, 2011.

[15] Y. Muroya, H. Li, and T. Kuniya, "Complete global analysis of an SIRS epidemic model with graded cure and incomplete recovery rates," Journal of Mathematical Analysis and Applications, vol. 410, no. 2, pp. 719-732, 2014.

[16] S. Kim, J. Byun, and I. Jung, "Global stability of an SEIR epidemic model where empirical distribution of incubation period is approximately by Coxian distribution," Advances in Difference Equations, vol. 469, 2019.

[17] M. Y. Li and J. S. Muldowney, "Global stability for the SEIR model in epidemiology," Mathematical Biosciences, vol. 125, no. 2, pp. 155-164, 1995.

[18] M. Fan, M. Y. Li, and K. Wang, "Global stability of an SEIS epidemic model with recruitment and a varying total population size," Mathematical Biosciences, vol. 170, no. 2, pp. 199-208, 2001.

[19] H. Zhang, L. Yingqi, and W. Xu, "Global stability of an SEIS epidemic model with general saturation incidence," International Scholarly Research Notices, vol. 2013, Article ID 710643, 11 pages, 2013.

[20] SUSAM, Secretaria de Estado de Saude do Estado do Amazonas, SUSAM, Manaus, Brazil, 2020, http://www.saude.am. gov.br/.

[21] L. López and X. Rodó, "A modified SEIR model to predict the COVID-19 outbreak in Spain and Italy: simulating control scenarios and multi-scale epidemics," SSRN Electronic Journal, 2020.

[22] Q. Li, X. Guan, P. Wu et al., "Early transmission dynamics in Wuhan, China, of novel coronavirus-infected pneumonia," New England Journal of Medicine, vol. 382, no. 13, pp. 1199-1207, 2020.

[23] Z. Wu and J. M. McGoogan, "Characteristics of and important lessons from the coronavirus disease 2019 (covid-19) outbreak in China," JAMA, vol. 323, no. 13, p. 1239, 2020.

[24] D. He, J. Dushoff, T. Day, J. Ma, and D. J. D. Earn, "Inferring the causes of the three waves of the 1918 influenza pandemic in England and Wales," Proceedings of the Royal Society B: Biological Sciences, vol. 280, no. 1766, p. 20131345, 2013.

[25] EPICOVID19, Epidemiologia da COVID-19 no Rio Grande do Sul, Boletim do Governo do Estado do Rio Grande do Sul, Porto Alegre, Brazil, 2020.

[26] B. Tang, X. Wang, Q. Li et al., "Estimation of the transmission risk of the 2019-nCoV and its implication for public health interventions," Journal of Clinical Medicine, vol. 9, no. 2, p. 462, 2020.

[27] N. Chen, M. Zhou, X. Dong et al., "Phase-adjusted estimation of the number of coronavirus disease 2019 cases in Wuhan, China," Cell Discovery, vol. 6, no. 1, 2020.

[28] Plotly Technologies Inc., Collaborative Data Science, Plotly Technologies Inc., Montreal, Canada, 2015, https://plot.ly.. 